\title{
SEPTIC POLYARTHRITIS BY HAEMOPHILUS INFLUENZAE
}

Caroline Diniz Bezerra ${ }^{1, \star}$, Leonardo Fernandes e Santana ${ }^{1}$, Mateus de Sousa Rodrigues ${ }^{1}$, Rita Marina Soares de Castro Duarte ${ }^{1}$

1.Universidade Federal do Vale do São Francisco, Petrolina (PE), Brazil.

*Corresponding author: carolinedinizbezerra@gmail.com

\section{BACKGROUND}

Septic arthritis is defined as intra-articular infection, commonly bacterial. There is potential joint destruction, if there is no prompt recognition and early institution of adequate antibiotic therapy. It is classified into gonococcal and nongonococcal. Among the latter, Staphylococcus aureus is the most common agent in adults, being rarely caused by Haemophilus influenzae. This case aims to alert to the peculiar clinical and epidemiological aspects of this pathogen.

\section{CASE REPORT}

J.F.L., 41 years old, male. Previously healthy, in custody, sent from a penitentiary. He reported arthritis in the ankles, knees and right elbow for 10 days associated with fever for 4 days. He denied use of legal or illegal drugs, denied also unprotected sex, urethritis, red eye or skin lesions. Upon examination: malnourished and febrile. There was arthritis in the ankles, right elbow and knees with active and passive flexion-extension of ankles and right elbow limited. Teeth were in bad status. Laboratory: Hb: 10.2 g/dL, leukocytes: 37,420/mm 3 ; lymphocytes: $1,497 / \mathrm{mm}^{3}$, platelets: $575,000 / \mathrm{mm}^{3}$. Hepatitis B and C, HIV, toxoplasmosis, cytomegalovirus and syphilis serologies were negative. The knees were punctured, with purulent aspirate, which was sent for analysis. After material was sent for culture (blood, urine, urethral secretion and pharynx), ceftriaxone $2 \mathrm{~g} /$ day, intravenously, and a single dose of azithromycin $1 \mathrm{~g}$, orally, were empirically started. Surgical drainage was performed on the knees, ankles and right elbow with drainage of purulent secretion. Synovial fluid (SF): glucose: 8 mg/dL, LDH: 43,711 U/L, protein: 3.3 g/dL. Bacterioscopy: gram-negative bacilli and coccoids. Blood cultures and SF culture identified Haemophilus influenzae. Urine culture and AARB SF: negative. Normal chest $X$-ray, echocardiogram and abdominal ultrasound. New surgical toilet was performed after 3 weeks. The patient progressed with clinical and laboratory improvement and was referred to the outpatient clinic, after 4 weeks of intravenous antibiotic therapy, with a schedule of 3 more weeks of oral antibiotic therapy.

\section{CONCLUSION}

Septic arthritis due to Haemophilus influenzae in adults was first described in 1938. Currently, Haemophilus influenzae subtype $\mathrm{B}$ arthritis is uncommon due to the routine protection by conjugate vaccines, while nontype $\mathrm{B}$ has been gaining epidemiological importance. Most cases have predisposing factors, such as diabetes mellitus, rheumatoid arthritis, alcoholism, trauma, among others, but the primary focus is not identified. It is associated with polyarticular presentation and frequent extra-articular involvement, making it necessary to screen other infectious focus, mainly in the upper airways. In this case, we did not identify predisposing factors or a concomitant infectious focus. 